\title{
Improvement of Mobility Management in Heterogeneous Wireless Networks by Using Multiple Attribute Decision Making
}

\author{
Mojgan Taheri (Corresponding author) \\ Student, Management Information Technology \\ Department of Management and Economics, Science and Research Branch \\ Islamic Azad University, Tehran, Iran \\ E-mail:mojgan423@yahoo.com \\ Mohammad Ali Afshar Kazemi \\ Faculty Member of Central Tehran Branch, Islamic Azad University, Tehran, Iran \\ E-mail:dr.mafshar@gmail.com \\ Abbas Toloie-Eshlaghy \\ Faculty Member of Science and Research Branch, Islamic Azad University, Tehran, Iran \\ E-mail: toloie@gmail.com
}

Received: June 4, 2011

Accepted: June 19, 2011

doi:10.5539/cis.v4n4p100

\begin{abstract}
Recently, there has been great interest in the community over heterogeneous wireless networks in order to support services with high quality. The integration of Wimax and Wi-Fi technologies in communication systems such as Voice ,FTP,Video conducted heterogeneous system and reveal that the proposed network selection technique can effectively decide the optimum network through making trade-offs among network condition, user preference while avoiding frequent handoffs. We identify vertical handover decision algorithm suitable for multimode mobile devices based on the Analytic Hierarchy Process (AHP) and evaluate its performance through simulation by Opnet.

This paper gives a comparative analysis of two queuing systems FIFO and WFQ by showing the advantages of using suitable weight which was calculated by AHP. The simulation results show that WFQ technique has a superior quality than FIFO. It could be opportunity to network designers and suppliers are providing the resources to servers more efficient use.
\end{abstract}

Keywords: WiFi,Wimax, Quality of Service (QoS), Analytic Hierarchy Process (AHP), First in First out( FIFO), Weight Fair Queue (WFQ)

\section{Introduction}

Today, advances in wireless communication technologies are driving the evolution toward ubiquitous and seamless service delivery across wireless systems. End users will access services via several existing access technologies such as General Packet Radio Service (GPRS), wireless local area network (WLAN), and Worldwide Interoperability for Microwave Access (Wimax).

Wimax is taken a relevant role in reaching the goal of realizing a full-service network all over urban and suburban area. At the same time, the wireless local area networks (WLAN) is widely used for in-home or short range communications, mainly basing on the Wi-Fi. The integration of Wimax and Wi-Fi technologies in communication systems requires the framework of intelligent vertical handoff decision algorithms to enable mobile users to seamlessly switch network access and experience uninterrupted service continuity anywhere and anytime by maintaining certain properties, mainly in terms of QoS.

People of the modern world are interested in using various network services like VOIP, Video conferencing, 
Email, Internet,... and File Transfer in every place and time. Different types of Traffic Management systems are used in those services. Each router in the network must implement some queuing disciplines that govern the packets are buffered while waiting to be transmitted.

Future mobile users will be always best connected anywhere and anytime via diverse access technologies and possibly in a Multi-operator of environment. This fact is posing unprecedented challenges to the resource management problem in such networks. In the competitive environment, the ultimate goal of resource management is maximizing provider's revenue.

Quality of services perceive in each network, including bandwidth, delay, Jitter and packet loss also cost are important in communication. User preferences in application are depend on the mentioned parameters.

Since wireless networks in technology access, network architecture, transport protocols, network coverage and other factors vary together, so different application has created various traffic in each of their users into the (first-come first serve) FIFO queue discipline which are entered in heterogeneous environment (Kerstin B. Johnsson, 2004). In this method all packets are treated equally by placing them into a single queue, then servicing them in the same order which they were placed in the queue. Delay and jitter will be increases in this method therefore other flows may be lost. (Gayathri Chandrasekaran, 2001).

According to the wireless network conditions that can only limited resources to address quality requirements to suit users allocate their preferences, mechanisms to allocate sufficient bandwidth available to different applications, to proportionate require quality of users.

Weighted Fair Queuing (WFQ) offers fair queuing that divides the available bandwidth between flows of traffic based on weights. Proper weight assigned to each queue and serves the higher weight queue which will be given rating priority in heterogeneous network. If the available bandwidth allocated to queue with lower priority is possible only if the queue of traffic there isn't a higher weight. (Binbin Chen and Mun Choon Chan, 2006)

Meanwhile, in order to combine multiple criteria together,weights are required to represent their relative importance, so certain weighting method, e.g. analytical hierarchy process (AHP), should be used to evaluate their weights. (Q. Y. Song and A. Jamalipour, 2005)

This survey article analyzes the most recent research efforts in area of queue theory and Multiple Attribute Decision Making, in integrated two wireless networks such as Wimax and Wi-Fi, attempting to allocate the appropriate weight which is calculated by AHP to the queue of users with different applications in order to priority queue WFQ is developed to provide quality services at minimum cost and delay also with maximum performance.

\section{Related Work}

Mobile users require wireless communication interface to facilitate connection to the internet, phone, video conference and other services. To this end management system for standards movement local wireless networks and wide wireless network have been proposed extensively. These systems based on different compounds to support mobility management and continuity in connect between networks. Characteristics such as bandwidth, delay, packet loss rate and cost in this decision take into consideration. Compare the performance of these networks decision vertical handoff by decision making techniques such as TOPSIS, SAW, GRA, MEW has been done. (Enrique Stevens-Navarro and Vincent W.S. Wong, 2006)

Analytical Hierarchy Process (AHP) AHP propose network selection scheme to weigh of QoS parameters. With these parameters, the authors construct an AHP hierarchy based on their relationships. QoS is placed in the topmost level as the objective, main QoS parameters is describing network condition such as availability, throughput, timeliness, reliability, security. The mechanism is divided into three main logical function blocks: collecting data, processing data and making decision. Service quality parameters based on user preferences and applications desired weight are given. Rating serve this purpose, users with a minimum time occurs. (Meriem Kassar, Brigitte Kervella, 2005)

Consequently, network selection techniques play a vital role in ensuring quality of service in heterogeneous networks. The proposed scheme comprises two parts, with the first applying an analytic hierarchy process (AHP) to decide the relative weights of evaluative criteria set according to user preferences and service applications, while the second adopts the obtained weight to users queue. In the simplest situation when the order entry queue user traffic based on demand reached earlier, their service will be done sooner. In fact, if a network or servers available for application to higher quality services that require access to ongoing support services were not in queue and wait time to spend equal.(Q. Song and A. Jamalipour ,2005)

While entering users by WFQ desirability serve more traffic queues under conditions of networks, the different 
applications require various performance which are more flexible. (Bertsekas and Tsitsiklis, 2002)

\section{Heterogeneous wireless networks}

Development of wireless communication networks to provide comprehensive integrated services is created. Users move typically to access network services by Global Mobile System (GSM), GPRS, Universal Mobile Telecommunications System (UMTS), Wi-Fi and WIMAX.

Mentioned networks with regard to how the extent of coverage and access are divided to wireless personal area network, local wireless networks, wireless Metropolitan Area Network, wide wireless networks and Satellite Network. The combination of different technologies provides the possibility of data services and high-quality media. (A. Salkintzis, 2004)

Recent studies show that Wide Wireless Networks like Wimax allows integration with Local Wireless Networks such as Wi-Fi for multimedia services and others are under the IP protocol. (Kandaraj Piamrat, C'esar Viho, Adlen Ksentini, Jean-Marie Bonnin, 2008)

The Wimax standard 802.16 to about $400 \mathrm{~m}$ radius and bandwidth $3.5 \mathrm{GHZ}$ with a maximum data rate $20 \mathrm{Mbps}$ and bandwidth 2.4 GHZ support users and under the standard 802.11 Wi-Fi cover services up to $80-70 \mathrm{~m}$ with a maximum radius bandwidth of 54Mbps and data rate at $4 / 2 \mathrm{GHZ}$.These two wireless technologies have common factors but the communication area are different.

Many access points in Wi-Fi are needed to support users as one base station of Wimax. Wi-Fi technology suitable for domestic use because of its coverage areas is short. While, Wimax is designed for long distances. Wi-Fi can support 12 users per access point, while Wimax can be up to 500 users at a base station supports. Wimax and Wi-Fi may combine their respective advantages on coverage and data rates, offering a high Quality of Service (QoS) to mobile users in heavy traffic. (H.Nmuyingi, T.Muchenje,2008)

In wireless communication network, each mobile user received several request which is encountered at any time and any where. Terminal decides whether communication through the same network must be done or should be connected to another network. Traditionally, the handoff process has been considered and studied among wireless networks using the same access technology (e.g., among cells of a cellular network) which is named horizontal handoff. Now, with the emerging mix of overlapped heterogeneous wireless networks deployed, the handoff has more complicated process. The handoff process among networks using different access technologies is defined as vertical handoff. (D. Chalmers and M. Sloman, current version2009)

\subsection{Quality of Service Mechanisms in Wireless Networks}

Quality of Services is based on the application, a set of service performance and the effect of determining the degree of user satisfaction in how to provide their service according to European Telecommunications Standards Institute. QoS parameters are including bandwidth, delay, jitter (delay variation), packet loss for delivery of network services such as voice, video conferencing and other application which can control by network administrators to provide users consent.

In case of same bandwidth is assigned to different applications, consequently service performance will be reduced. Allocation of service providers according to the level of traffic and service quality may be differed. For example, voice requires low bandwidth, but the lack of packet, low delay and jitter could be little. Users of video accept lack of some packets, but the delay and jitter is not acceptable. Internet traffic often has the lowest priority because of the connection through with minimal delay is approved and it can accept the lack of packages. Multimedia has shorter delay and needs bigger bandwidth. Applications such as e-mail do not sensitive to delay and bandwidth but lack of packet is important. But Real-time applications like video conferencing are sensitive to delay and require a lot of bandwidth. (Y.-B. Lin,1997)

On the other hand moves between two different networks need to recognize and permit when moving from one network to another network in a minimum delay. Satisfaction of users will be achieved in lowest possible delay in services and highest performance.

\subsection{Service to users queue}

Queuing Theory of many networks can be modeled by a set of lines where users arrive with a certain rate and leave the network in another rate. Queuing is the way of traffic packets are lined and waited for processing by the scheduler. The scheduler decides which queue and packets should be transmitted. The order in which the scheduler selects the packets to process can effect to network performance.

The main characteristics of queue process is order entry that often serve as receptors in a single specific time or average time between two continues arrival are expressed. The arrivals of new request into a cell in 
heterogeneous network depend on arrival distribution which are considered different. Application requirements can be different according to parameters such as bandwidth and delay. Rating service rate $\mu$ and arrival rate $\lambda$ in customer networks would be varied. (Enrique Stevens-Navarro and Vincent W.S. Wong, 2008)

Exponential distribution in wireless networks is more common. For instance, the conversational applications often prefer to pay less cost therefore the exponential distribution model fitted well for the time of serving .Applications such as file transfer, because the size of a large variance hypo-exponential distribution can be spent time in the service can be used. (M. Li, M. Claypool, R. Kinicki, and J. Nichols, 2005)

In heterogeneous environment, the nature and characteristics of each network are different therefore generally can not fixed rate for the distribution of arrival and time for service handoff .It may assume that the arrival rate of new user follows a Poisson process for application such as voice ,date and duration of a service is assumed exponentially distributed.( Faouzi Zarai,2008)

For our purpose, the exponential process is considered to be a reasonably good approximation since we are dealing with a connection-level admission control and resource management.

\subsubsection{Method of Weight fair queue}

Weighted Fair Queueing (WFQ) offers fair queueing that divides the available bandwidth between flows of traffic based on weights. It is one of dynamic allocation method in providing quality services which is with increasing traffic or changing application, the queue weights are updated. (U.Fiedler, 2001) The bandwidth would be divided unequally in many services in networks. For instance, when a type of real-time traffic with data traffic should be served, real-time traffic requires less bandwidth. In such cases the specific weighting for each line is used and the amount of dedicated bandwidth to each connection based on the weighting is determined. (T. Ahmed, K. Kyamakya, and M. Ludwig, 2006)

In this research, decision criteria are based on applications and wireless network profile. Queue is weighted by using AHP which will be set manually.

\section{Weight to queue of users by AHP technique}

Thomas L. Saaty develops a system called AHP (figure1) that transforms the pairwise comparison scores into weights of different attributes and priorities of all alternatives on each attribute to given weight assignment.

The procedure of AHP can be summarized as: 1) formulate the problem; 2) determine the relative weights of the comparison attributes; 3) compare the alternatives on each attribute; and 4) aggregate weights to produce final evaluation.(V. K. Thomas ,2007)

In the delivery of packets among networks with different technologies suitable assignment is depended on consent of users which is appropriate by AHP model according to Kerstin B. Johnsson (2004). Applying of AHP in decision making is based on user preferences and user application to assign respective weights. In network selection, users try to service in any place and any time through optimized network is chosen. Investigate the service quality at the highest level of the hierarchy to select the network, so confident in quality of service profile will be the target selection process network.

AHP has been selected because the ability to create various weights between each target. It is allowed the user to solve complex problem by instructing ideas hierarchically and then perform the paired comparison of the elements. Decision making parameters have been considered such as network conditions (bandwidth, delay, jitter and packet loss and the costs of wireless network) and user preferences in services (applications in real time and non real) . Based on priorities by users, scores between 1 to 9 was assigned, while score 1 with the highest preference and the lowest is 9. (T. Ahmed, K. Kyamakya, and M. Ludwig, 2006)

\section{Proposed model}

Wireless communication system environment with different technologies in applications such as voice, video and data are considered and users are on the move between local and wide wireless networks. Mobile users have possibility to obtain their services from different type of technology in some parts of environment .Movement between networks depend on the quality of service requirements which are necessary to create a heterogeneous networks .Users pass into heterogeneous environment under exponential distribution with hypothetical rate to achieve their service.

Typically schedule algorithm will be FIFO which is allocated equal bandwidth to the ability to assign users with minimum bandwidth to each user and avoid packet loss has to be arrived.

While if we consider users arrive under WFQ discipline, it will be possible to assign suitable weight to users 
stations with different applications that are far from serving. Weights are considered based on user preferences and access network profile which is calculated by AHP.

The parameters are determined based on the importance of each criteria such as cost, bandwidth, delay, jitter, and packet loss based on user preferences are assessed .Available networks are evaluated in comparing mentioned indicators . Voice, video services (real time service) and data transfer (non-real time service) are studied.

Proper weight for each indicators in handoff between Wi-Fi and Wimax are assigned and their average are determined by AHP. Allocated weights to each queue can varied and determined the amount of resource that the router are allow to assign to desired queue, it remains constant during the time of connection.

In Figure 2 observed conceptual model how weight of WFQ scheduling that calculated by AHP is assigned to base stations of Wimax or access point of Wi-Fi. Therefore queue of users with different application will be served under WFQ.

\subsection{Calculation of weights by AHP}

Users arrive in heterogeneous environments, appropriate network of those that are available, based on user preferences are selected in minimum delay and maximum throughput. If suitable network is not selected for service to user when are moving between Wimax, base stations and Wi-Fi , access points handoff may occur several times ,therefore delivery operation lead may be disconnected. Unrealistic and non-useful of delivery operations will be cause of reducing quality of service. Service delivery for better communication means that not only communicates but also in the best possible quality service at any time and place should be done.

Gathered information such as user preferences and network conditions as input to the decision algorithm have been collected by distributing questionnaires among users and experts of network design. QoS parameters in each application associated with the goal of best quality, are varied. Therefore, these parameters and cost for different applications with lower and higher values are compared .(Q. Song and A. Jamalipour, 2005)

The fundamental scale for pairwise are considered as follows:

- Intensity of importance of 1 is defined as equal importance

- Intensity of importance of 3 is defined as moderate importance

- Intensity of importance of 5 is defined as strong importance

- Intensity of importance of 7 is defined as extreme importance

Table 1 shows preferences of user in application such as file transfer based on pairwise comparison matrix of cost and Qos parameters. An element in the matrix is 1 when it compare with itself and the other element depend on user preference .User ask themselves how much application of FTP is an average of all elements. As general rule, each element listed in left hand column is compared with the element of top row .If the comparison is important the cells fill in with and integer and in the opposite case the judgment is a fraction.

For combining multiple criteria together, the weight is needed to evaluate that AHP technique is used . For each matrix, reasonable adjustment is also evaluated. If a combination of voice and video applications or other application were desired by mobile user, combined weights should be calculated.

The output of the process are the overall preferences for the five elements is solved using the geometric mean method over each column which results are shown in table 2.As it can be observed lack of parcel is important for FTP's user because its weight is 0.487 .

With the calculated relative scores the priorities (i.e. weights) for the 5 objectives in terms of the overall goal in each application of voice, FTP and video is obtained.

Table 3 is compared of Wi-Fi and Wimax in view of Bandwidth .For selecting a suitable network pairwise comparison matrixes for each parameters are formed.

Preference matrix are normalized and determined with the average weight of each network AHP technique to different applications in the indices. Arithmetic mean of weights of FTP, video and audio is calculated in table 4.

Network with the highest score is selected for each application. For instance user with application of video prefers to select Wimax and Wi-Fi for FTP is chosen.

\section{Simulation of model in Opnet:}

Simulation model in Opnet Software oriented simulation language that can be done. The OPNET model library provides several queuing mechanisms that are commonly used in network routers and switches. These include First In First Out (FIFO) and Weighted Fair Queuing (WFQ).

In this research, heterogeneous environment, consisting of several Wimax base stations, Wi-Fi access points and 
routers based on QoS parameters are considered. In mobile communication, when users of the previous station want to the next moves, the current relationship between the user's previous base station and mobile base stations in the next delivery, should be preserved. We compare deferent queue technique, incoming packets which is lined based on FIFO with WFQ that the weight of the user preferences was assigned according to AHP technique. Different parameters of QoS are considered and user preferences based on application of sound, Video and file transfer are investigated. FIFO, WFQ purpose actually served a balance between complexities is forming. (figure 3)In this study, comparing these two discipline where considered in the available networks and applications to change, forming the best service is done. Process queue weights setting time intervals specified by the network administrator of the system. Minimizing delay and try to make the maximum performance is target in service to users.

\section{Evaluation of the proposed method}

Users with any application, prefer that in minimum delay are served. The weight from applying preferences of users by AHP technique in figure 4 is observed that delay in arrival of users in comparison of FIFO and WFQ is lower.

During the integration and interaction in vertical handoff between Wi-Fi and Wimax technology performance compared for FIFO and WFQ. In Figure 5 and 6 observed that WFQ offers a more optimal state as more packed during time can be served.

\section{Conclusion}

Wi-Fi and Wimax networks have different characteristics in flow of traffic management and quality of services. Access point of Wi-Fi communicates with base station of Wimax in the simulation. Mobility of users in a wide area and access anywhere will be guarantee in Wimax, while we have limited access in Wi-Fi under places near to access points. Using Wi-Fi can support users at lower cost .On the other hand, Wimax with high access rate that is causing network integration can be support needs of users . Apparently, Wimax and Wi-Fi technologies would be as complements.

Combining of two technologies satisfactory performance for users is increased in more optimal use of network traffic when loaded. Coverage of Wimax in heterogeneous environments and some access point in area of Wi-Fi would be a solution to communicate across.

To cover wireless networks would be ideal choice for users on application of FTP, voice and video. Moreover, the combination of wireless technologies such as Wimax and Wi-Fi is possible solution for high speed communications. Even it is effective in cheapest price of components for communications in local and wide area.

The obtained results in delay and performance could be used for network designers to practice as a strategy for the performance of network. Also it will be usable for Internet providers to adjust application parameters to achieve optimal levels of service quality in heterogeneous wireless networks. The result may show that bandwidth available for allocation among the queues of traffic can be classified as appropriate.

Simulation results show that if the traffic queues are weighted quality requirements of voice and video services order entry management WFQ are more regular. End to end delay, jitter is lower thus quality of services provided by WFQ very appropriate comparing with FIFO.

\section{References}

Kerstin B. Johnsson. (2004). Quality of service scheduling for heterogeneous data services on wireless systems, A dissertation submitted to the Department of Electrical Engineering and the committee on graduate studies of Stanford University in partial fulfillment of the requirements.

Binbin Chen and Mun Choon Chan. (2006). Resource Management in Heterogeneous Wireless Networks with Overlapping Coverage, COMSWARE, New Delhi ,Department of Computer Science National University of Singapore.

Enrique Stevens-Navarro and Vincent W.S. Wong. (2006). Comparison between Vertical Handoff Decision Algorithms for Heterogeneous Wireless Networks, VTC. IEEE $63^{\text {rd }}$ Volume: 2 DOI. 10.1109/VETECS.2006.1682964 Page(s): 947 - 951.

Meriem Kassar , Brigitte Kervella. (2008). An overview of vertical handover decision strategies in heterogeneous wireless networks, Guy Pujolle University Pierre \& Marie Curie ,France Computer Communications 31 (2008) 2607-2620Elsevier doi:10.1016/j.comcom.2008.01.044.

Q. Song and A. Jamalipour. (2005). An adaptive quality-of-service network selection mechanism for 
heterogeneous mobile networks, Wireless Communications and Mobile Computing, vol. 5, no. 6, pp. 697-708, Bertsekas and Tsitsiklis. (2002). Introduction to Probability Athena Scientific. http://www.athenasc.com/probbook.html

A. Salkintzis. (2004). Interworking Techniques and Architectures for WLAN/3G Integration Toward 4G Mobile Data Networks, Wireless Communications, IEEE, vol. 11, no. 3, pp. 50-61, DOI: 10.1109/MWC.2004.1308950.

Kandaraj Piamrat, C'esar Viho, Adlen Ksentini, Jean-Marie Bonnin. (2008). Resource Management in Mobile Heterogeneous Networks: State of the Art and Challenges Theme COM Systemes communicants Equipe-Projet Dionysos Rapport de recherche n 6459.

H.Nmuyingi, T.Muchenje. (2008). An Overview of Security Issues on a Converged Wi-Fi and WiMAX Network. Tonderai Muchenje, Hippolyte Muyingi Telkom Centre of Excellence (CoE) in Developmental E-Commerce. Computer Science Department University of Fort Hare, Alice, P.Bag X1314, 5700 South Africa.

D. Chalmers and M. Sloman. (1999, current version 2009). A Survey of Quality of Service in Mobile Computing Environments, IEEE Commun. Tutorials and Surveys, 2nd qtr. 1999.

European Telecommunications Standards Institute, General aspects of quality of service and network performance in digital networks, including ISDN, Technical report ETR 003 ed.1, ETSI, 1990.

Y.-B. Lin. (1997). Performance modeling for mobile telephone networks, IEEE Network, pp. 63-67.

Enrique Stevens-Navarro and Vincent W.S. Wong. (2008). Virtual Partitioning for Connection Admission Control in Cellular/WLAN Interworking", Wireless Communications and Networking Conference, 2008. WCNC 2008. IEEE , DOI: 10.1109/WCNC.2008.362

H. Dewing, S. Potter. (2002). Implementing QoS solutions in enterprise networks, URL reference.

M. Li, M. Claypool, R. Kinicki, and J. Nichols. (2005). Characteristics of streaming media stored on the web. ACM Trans. Inter. Tech., vol. 5,no. 4, pp. 601-626.

Faouzi Zarai. (2008). Quality of Service and Security in Wireless Fourth Generation Networks. Prepared by CN\&S Research Lab. The Communication Network and Security (CN\&S) research Laboratory.

(University of 7th of November at Carthage, Tunisia). Approved for public release.

H.Wang C.Shen and K.Shin. (2001). Adaptive -Weighted packet scheduling for Premium Service, Proc .IEEE ICC 2001.

U.Fiedler. (2001). Porting a WFQ Scheduler into NS-2 Diffserv Environment, student Thesis, Eidgenossische Technice Hochschule Zurich Swiss Institute of Technology Zurich.

T. Ahmed, K. Kyamakya, and M. Ludwig. (2006). A context-aware vertical handover decision algorithm for multimode mobile terminals and its performance. in Proceedings of IEEE/ACM Euro American Conference on Telematics and Information Systems (EATIS '06), pp. 19-28, Santa Marta, Colombia.

T. L. Saaty. (1990). How to make a decision: The Analytic Hierarchy Process, European Journal of Operational Research, 1990, Vol. 48, pp. 9-26.

V. K. Thomas. (2007). PhD dissertation, Georgia Institute of Technology.

Q. Y. Song and A. Jamalipour. (2005). Network selection in an integrated wireless LAN and UMTS environment using mathematical modeling and computing techniques. IEEE Wireless Commun., vol. 12, no. 3, pp. $42-48$.

Table 1. pairwise comparison matrix

\begin{tabular}{|l|l|l|l|l|l|}
\hline FTP & cost & Bandwidth & Delay & Packet loss & Jitter \\
\hline cost & 1 & 3 & 3 & $1 / 3$ & 7 \\
\hline Bandwidth & $1 / 3$ & 1 & 1 & $1 / 5$ & 5 \\
\hline Delay & $1 / 3$ & 1 & 1 & $1 / 5$ & 5 \\
\hline Packet loss & 3 & 5 & 5 & 1 & 7 \\
\hline Jitter & $1 / 7$ & $1 / 5$ & $1 / 5$ & $1 / 7$ & 1 \\
\hline
\end{tabular}


Table 2. Calculation of criteria weights for FTP by AHP

\begin{tabular}{|c|c|c|c|c|c|c|c|c|}
\hline & $\begin{array}{l}8 \\
8 \\
0\end{array}$ & 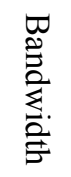 & $\begin{array}{l}\underset{\odot}{\stackrel{\vartheta}{2}} \\
\stackrel{2}{2}\end{array}$ & 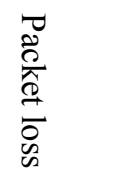 & 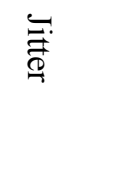 & $\stackrel{\longrightarrow}{\grave{X}}$ & $\begin{array}{l}\pi \\
\stackrel{0}{0} \\
\stackrel{0}{0}\end{array}$ & 节 \\
\hline cost & 1 & 3 & 3 & 0.3333 & 7 & 20.9979 & 1.83838 & 0.256 \\
\hline Bandwidth & 0.3333 & 1 & 1 & 0.2 & 5 & 0.3333 & 0.802726 & 0.112 \\
\hline Delay & 0.3333 & 1 & 1 & 0.2 & 5 & 0.3333 & 0.802726 & 0.112 \\
\hline Packet loss & 3 & 5 & 5 & 1 & 7 & 525 & 3.499708 & 0.487 \\
\hline Jitter & 0.1428 & 0.2 & 0.2 & 1 & 0.1428 & 0.000816 & 0.241159 & 0.034 \\
\hline & & & & & & & 7.184698 & 1 \\
\hline
\end{tabular}

Table 3. Comparing Wi-Fi and Wimax in view of Bandwidth

\begin{tabular}{|l|rrrll|}
\hline bandwidth & Wi-Fi & wimax & rjk & Root(5) & AHP \\
wifi & 1 & 5 & 5 & 2.236068 & $\mathbf{0 . 8 3 3}$ \\
wimax & 0.2000 & 1 & 0.2 & 0.447214 & $\mathbf{0 . 1 6 7}$ \\
& & & & 2.683282 & 1 \\
\hline
\end{tabular}

Table 4. The average weights of FTP, video and audio

$\begin{array}{llll} & \text { FTP } & \text { Video } & \text { Voice } \\ \text { Wifi } & 0.7361 & 0.3691 & 0.1986 \\ \text { Wimax } & 0.2697 & 0.6319 & 0.7994\end{array}$

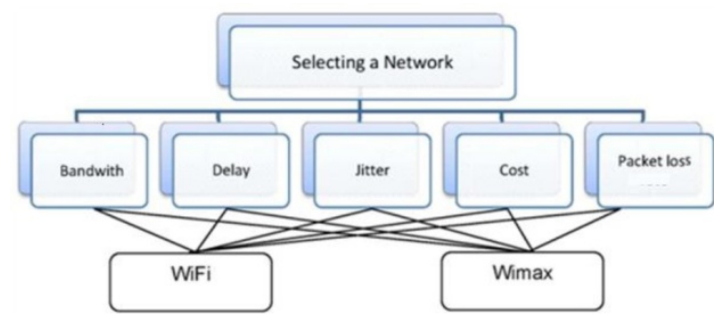

Figure 1. AHP

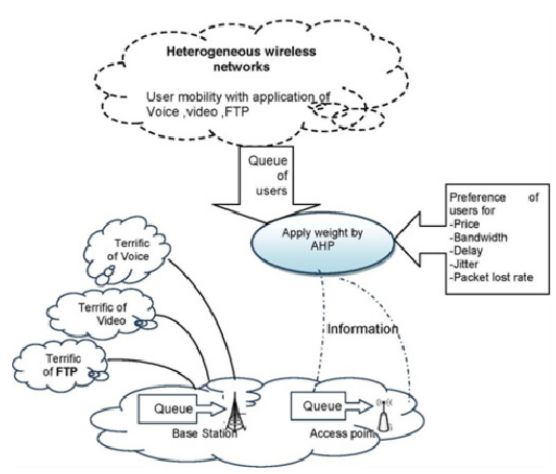

Figure 2. Context model of wireless communication in heterogeneous environments 


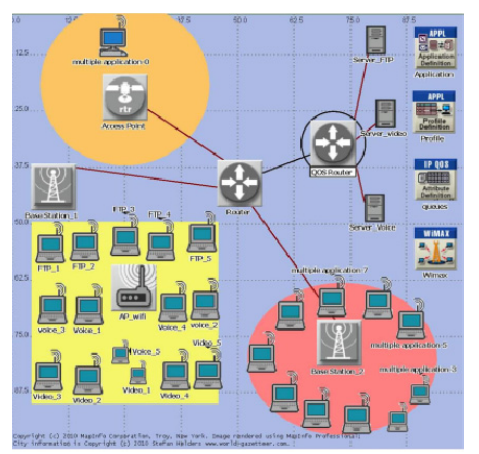

Figure 3. Simulation model user mobility in heterogeneous environments Opnet

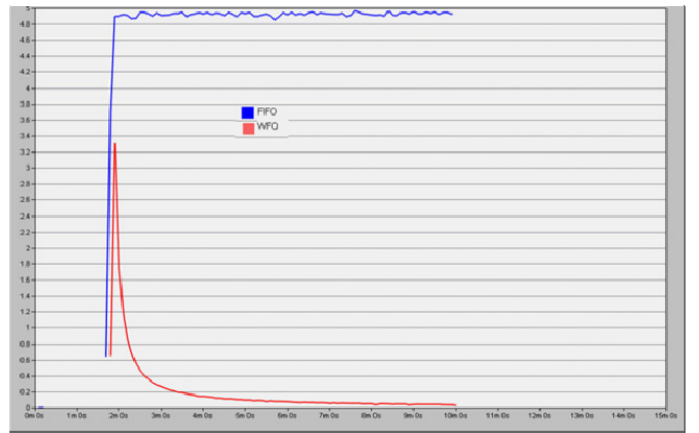

Figure 4. Comparison of delay in FIFO and WFQ

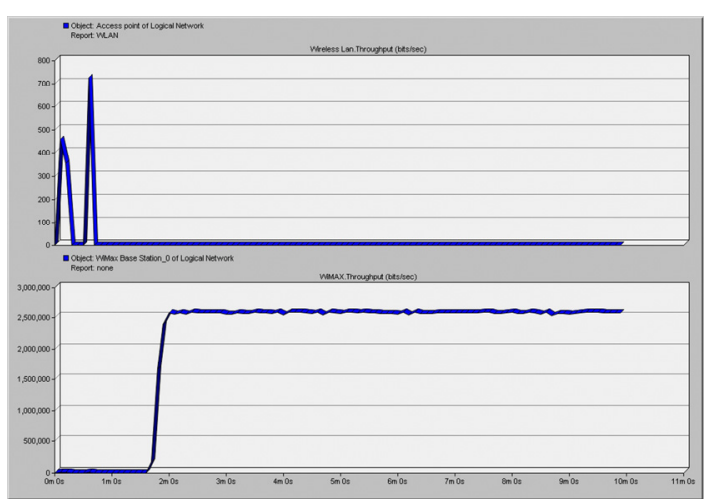

Figure 5. Performance service under FIFO discipline

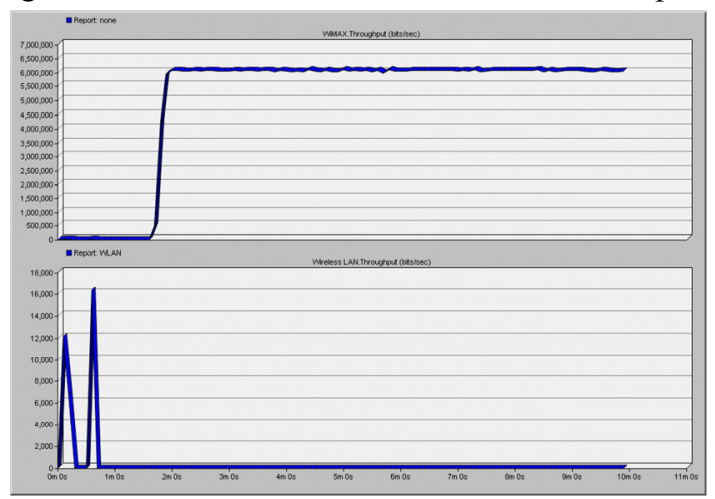

Figure 6. Performance service under WFQ discipline 